\title{
Preparation and characterization of sodium lauryl ether sulphate modified layered double hydroxides
}

\begin{abstract}
Layered double hydroxides (LDHs) has attracted much attention of many industrialists as well as academicians as these materials can be used as catalysts, adsorbents, anion exchangers, stabilizers and fillers for polymers. The materials which consist of divalent and trivalent cations, and anions are nontoxic and easy to prepare. In addition, the exchangeability of their anions makes them easy to be modified to increase their hydrophobicity property through anion exchange process using long chain surfactant anions. In this study, sodium lauryl ether sulphate (SLES) modified LDHs was prepared by coprecipitation of LDHs and followed SLES ions modification via ion exchange process. Intercalations of the anions into the LDHs layers were confirmed by X-ray diffraction analysis. The presence of functional groups before and after modification was studied by FTIR spectrometry. Thermalgravitmeric analysis also was carried out to determine the amount of surfactant intercalated into LDHs.
\end{abstract}

Keyword: Co-precipitation; Layered double hydroxides; Sodium laurylether sulphate 